\title{
Association of Insomnia in Patients with Chronic Kidney Disease on Maintenance Hemodialysis
}

Omer Farooq Rehman ${ }^{1}$, Uzma Rauf ${ }^{2}$, Maryam Rauf ${ }^{3}$, Sana Aziz ${ }^{4}$, Ahmad Faraz ${ }^{5}$, Farah Anum Jameel ${ }^{6}$

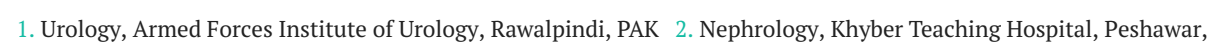
PAK 3. Plastic Surgery, Burns and Plastic Surgery Unit, Hayatabad Medical Complex Medical Teaching Institute (HMCMTI), Peshawar, PAK 4. Internal Medicine, Hayatabad Medical Complex (HMC), Peshawar, PAK 5. Trauma and Orthopaedics, Leeds Teaching Hospitals NHS Trust, Leeds, GBR 6. Nephrology, Jinnah Postgraduate Medical Centre, Karachi, PAK

Corresponding author: Omer Farooq Rehman, farooq.rehman@live.com

\section{Abstract \\ Objective}

To determine the frequency of insomnia in patients with chronic kidney disease (CKD) on maintenance hemodialysis (HD)

\section{Study design}

Cross-sectional (descriptive)

\section{Study duration}

From July 20, 2019, to January 20, 2020

\section{Study settings}

Department of Nephrology, Khyber Teaching Hospital, Peshawar

\section{Materials and methods}

A total of 148 patients diagnosed with CKD and on maintenance HD were selected for the study in a consecutive sampling manner and checked for insomnia.

\section{Results}

Out of 148 patients included in the study, there were $64.9 \%$ male and $35.1 \%$ female patients. The mean duration of CKD (months) was $13.9 \pm 6.3$. The mean number of sessions for hemodialysis done in all patients was $16.8 \pm 5.3$. On careful interviewing of the patient, difficulty in falling asleep was recorded in $28.4 \%$,

Received 07/19/2020

Review began 07/20/2020 Review ended 07/20/2020 Published 08/02/2020

\section{(c) Copyright 2020}

Rehman et al. This is an open access article distributed under the terms of the Creative Commons Attribution License CC-BY 4.0., which permits unrestricted use, distribution, and reproduction in any medium, provided the original author and source are credited. difficulty in staying asleep in $41.9 \%$, problems in waking up early in $34.5 \%$, and insomnia interfering in routine life activities in $28.4 \%$. Overall, insomnia was recorded in $36.5 \%$ of patients and was found to have a significant correlation with the number of dialysis sessions (p-value 0.000).

\section{Conclusion}

Insomnia is a frequent disorder associated with CKD patients on maintenance HD. There are very few studies establishing its pathogenesis and risk factors. We recommend further multicenter studies to detect the course of insomnia in association with CKD on HD and its potential impact on the overall quality of life of patients with CKD.

Categories: Psychiatry, Nephrology, Public Health

Keywords: chronic kidney disease, anxiety, insomnia, hemodialysis

\section{Introduction}

Patients with kidney disorders are blighted with various types of sleep problems. Many a time, healthcare professionals fail to recognize the kind of sleep disorder unless they know the common sleep-related issues in accordance with chronic kidney disease (CKD) [1]. Various sleep disorders are associated with CKD, which include insomnia, sleep-related breathing disorders, hypoventilation, central sleep apnea, central disorders of hypersomnolence, circadian rhythm sleep-wake disorders, parasomnias, and sleep-related movement disorders [2].

American Academy of Sleep Medicine (AASM) defined insomnia as a repeated difficulty with sleep initiation, duration, consolidation, or quality that happens despite adequate opportunity and circumstances for 
sleep and results in some form of daytime impairment [3]. Patients with insomnia have been observed to have problems with falling asleep, continuing sleep cycle, and/or unwanted sustained periods of wakefulness during sleep [4]. Studies have shown cognitive and physiological risk factors for Insomnia. Maung et al. found that metabolic disturbances secondary to chronic kidney disease may influence abnormalities in sleep behavior [5]. Patients with renal disorders, CKD, or end-stage renal disease (ESRD) are associated with progression to various sleep abnormalities.

Various studies show the impact of kidney dysfunction on sleep. Hanly et al. observed that there is a direct linear correlation between CKD or ESRD and insomnia. Specifically, a worsening CKD is directly associated with worsened insomnia [6]. Furthermore, a descriptive study published by Aggarwal et al. [7] observed insomnia among $60 \%$ of patients who underwent hemodialysis (HD), whereas other studies report the frequency of insomnia as $86.5 \%$ [8] and $45 \%$ [9].

To further describe the specific etiology, Rayner proposed a direct relationship between decreased orexin levels to a decline in wakefulness (narcolepsy) [10]. He further proposed that in the case of CKD or end-stage renal disease (ESRD) levels of orexin are significantly high, causing lost sleep [11]. Also, psychiatric overtones (depression, psychosis, and insomnia) can ensue due to hypercalcemia associated with CKD or ESRD [12].

The previously published study shows a variable proportion of insomnia in CKD but also gives a mixed type of sample size used [5]. Therefore, the current study has been set up to investigate the magnitude of insomnia among patients with co-morbid of CKD or ESRD on hemodialysis in our ethnic groups. We will request further studies over the usefulness of interventions designed to alleviate sleep disorders in these patients.

\section{Materials And Methods}

This cross-sectional (descriptive) study was conducted at the nephrology department of Khyber Teaching Hospital, Peshawar. Following approval from the hospital ethical and research committee, this study was conducted over a period of six months, from July 2019 till January 2020.

The sample size was $148,42.9 \%$, i.e., 15 proportion of insomnia in CKD patients on hemodialysis with a $95 \%$ confidence level and an $8 \%$ margin of error using the World Health Organization (WHO) sample size calculator. The sampling technique was non-probability consecutive sampling.

\section{Inclusion criteria}

CKD patients who underwent a minimum of 10 hemodialysis sessions

\section{Exclusion criteria}

1. Patients previously diagnosed with insomnia, anxiety, or depression

2. Patients with a history of emotional trauma, sudden loss of assets, or in a near relative, in the previous six months

All patients meeting the selection criteria were included in the study through the outpatient department (OPD) or the dialysis center. Written informed consent was taken from all the patients and they were subjected to a detailed history and clinical examination and underwent an in-depth interview in an undisturbed environment to detect findings suggestive of insomnia. Findings were recorded in the presence of a consultant nephrologist and an attending psychiatrist, with both having a minimum of five years of consultancy experience.

After recording the necessary demographics, care was taken during the extraction of information from all patients to avoid responder bias. Confounders and other biases were controlled by strictly following the exclusion criteria.

Data were recorded in Statistical Package for the Social Sciences (SPSS) version 20 (IBM Corp, Armonk, NY). Descriptive statistics were used to analyze data, where mean and standard deviation was calculated for quantitative variables like age, duration of CKD, and the number of hemodialysis sessions (HDs) done in the past. Whereas, frequency and percentages were calculated for categorical variables (gender and insomnia). Insomnia was stratified according to the age, gender, duration of CKD, and episodes of hemodialysis, to establish the effect modifiers using the chi-square test with a P-value of $<0.05$ as significant and the independent-sample t-test used for comparing means between the groups.

\section{Results}

A total of 148 subjects were included in the present study, which had CKD as per the operational definition. Their demographic and clinical data have been depicted in Table 1. The age of the included patients ranges 


\section{Cureus}

between 18 and 60 years. Male outnumbered females (64.9\% vs 35.1\%) in our study. Age did not impact insomnia with a p-value of 0.362 , whereas gender did not show any significant correlation (p-value 0.713 ) as well, as given in Table 1. After applying chi-square, we found no significant association between insomnia and the duration of CKD (p-value 0.940).

\begin{tabular}{|c|c|c|c|}
\hline No. of patients & 148 & \multicolumn{2}{|l|}{ p-value } \\
\hline \multicolumn{4}{|l|}{ Gender } \\
\hline Male & $96(64.9 \%)$ & \multirow{2}{*}{0.713} & \\
\hline Female & 52 (35.1\%) & & \\
\hline \multicolumn{4}{|l|}{ Age range } \\
\hline 18 to 30 years & 26 & \multirow{4}{*}{0.713} & \\
\hline$>30$ to 40 years & 47 & & \\
\hline$>40$ to 50 years & 31 & & \\
\hline$>50$ to 60 years & 44 & & \\
\hline \multicolumn{4}{|c|}{ Newly diagnosed patients of Insomnia on hemodialysis } \\
\hline \multicolumn{4}{|l|}{$54(36.5 \%)$} \\
\hline \multicolumn{4}{|l|}{ Hemodialysis sessions } \\
\hline 11-15 & 81 & & \\
\hline$>15-20$ & 12 & & \\
\hline$>20-25$ & 55 & & \\
\hline DURATION OF CKD & With insomnia & Without insomnia & \multirow{4}{*}{0.940} \\
\hline 3 to 9 months & 15(38.5\%) & $24(61.5 \%)$ & \\
\hline$>9$ to 16 months & $18(36.7 \%$ & $31(63.3 \%)$ & \\
\hline$>16$ to 24 months & $21(35.0 \%)$ & $39(65 \%)$ & \\
\hline
\end{tabular}

TABLE 1: Demographic characteristic of patients

Insomnia fulfilling the International Classification of Sleep Disorders-2 (ICSD-2) criteria [12] was found among 54 patients (36.5\%). On further questioning the subjects, we found that difficulty in initiation of sleep was recorded among $28.4 \%$, problems in maintaining sleep state in $41.9 \%$, problems in waking up early in $34.5 \%$, and sleep deprivation interfering in routine life activities in $28.4 \%$. We stratified insomnia with regards to different age groups, gender, duration of CKD, and the number of hemodialysis episodes. The Fisher-exact test suggested that insomnia was associated with hemodialysis ( $p$-value 0.000). However, it did not show any significant correlation with the age of the subjects, and the p-value was found to be 0.362 (Table 2). 


\section{Cureus}

\begin{tabular}{|l|l|l|}
\hline Age Group Category & Patients with Insomnia & p-value \\
\hline 18 to 30 years & $9(34.6 \%)$ & 0.396 \\
$>30$ to 40 years & $19(65.4 \%)$ & \\
$>40$ to 50 years & $14(45.2 \%)$ & \\
$>50$ to 60 years & $12(27.3 \%)$ & \\
HEMODIALYSIS & & 0.000 \\
$11-15$ & $81(54.7 \%)$ & \\
$>15-20$ & $12(8.1 \%)$ & $55(37.2 \%)$ \\
$>20-25$ & & \\
\hline
\end{tabular}

TABLE 2: Correlation of age group and hemodialysis sessions with insomnia

\section{Discussion}

The present study suggests that insomnia can be frequently observed among patients with CKD. Nearly onethird of our patient group were diagnosed with insomnia, whereas several studies have shown a wide range of prevalence ranging from $54 \%$ to $69 \%$. Therefore, the need to adopt more effective treatment strategies for CKD insomniacs is of paramount importance [13].

Murtagh et al. published a systemic review of 17 studies, where he reported $44 \%$ of the patients had sleep disturbances with underlying co-morbid ESRD [14]. The purpose of the current study is to highlight the significance of insomnia and other sleep-related conditions because they are under-recognized by renal healthcare providers as reported by Weisbord et al. in his descriptive study [15].

Sabbatini et al. found a higher prevalence of insomnia among females and elderly patients [16]; this is in contrast to our study, as we report a higher number of patients with insomnia in the age group of 30-50 years. The most common reason for insomnia in our study was difficulty in staying asleep and early morning awakening, whereas Shahbaj et al. reported problems in sleep initiation and sleep maintenance as more prevalent causes for Insomnia [17].

CKD is associated with diabetes, depression, and increased cardiovascular mortality. Consequently, insomnia ensues secondary to diabetes and depression [18]. Insomnia in CKD subjects could be due to various factors: money spent on therapy, co-morbid sleep disorders like restless leg syndrome (RLS) and obstructive sleep apnoea, and risk factors such as diabetes and anxiety [19]. However, we did not investigate the financial burden among subjects; it could be one of the primary reasons for insomnia among third-world countries.

Diabetes and cardiovascular diseases are significant causes of insomnia, as reported by Manan et al. [17], but we could not assess causality due to the cross-sectional nature of the study. A descriptive study published by Mark et al. assessed sleep quality among patients with insomnia and reported three times more risk to sleep less than five hours per night and more than half had difficulty getting back to sleep or waking up too early in the morning or feeling tired and not getting ample amount of sleep [20]. In addition, he reported insomnia in 50\%-80\% of patients on hemodialysis; the current study observed a similar trend between insomnia and hemodialysis. Rai et al. validated these findings by proposing that insomnia was present in $60.9 \%$ of patients on hemodialysis and significantly higher in patients on dialysis for more than one year $(\mathrm{p}=0.003)[21]$.

Sleep-related problems are often overlooked by physicians and often omitted by the patient due to various reasons such as negative approach by the family or patient towards psychiatric illnesses, lack of interdisciplinary communication and cooperation among medical specialties [22]. Melatonin is recommended for the regulation and improvement of the sleep-wake cycle in patients with insomnia, as it improves the subjective and objective parameters of sleep, with no consequent side effects [23]. Yang et al. reviewed around 12 randomized controlled trials (RCT) and one cohort study to analyze the effect of nonpharmacological treatment modalities (cognitive-behavioral therapy (CBD), acupressure, exercise) with insomnia, and concluded that $\mathrm{CBD}$ for insomnia is a recommendable treatment option for patients on hemodialysis [24].

\section{Limitations}


The limitations of our study include it being a single-center study with limited sample size. Information regarding the quality of life and financial aspects were not included in the study. Behavioral factors as causes of insomnia and their consequences on the quality of life were not reviewed. Furthermore, we did not evaluate various co-morbidities among CKD patients and did not investigate other sleep-related problems such as obstructive sleep apnea (OSA) and parasomnia.

\section{Clinical recommendations}

There is a need for practicing physicians to address sleep complaints in their patients with chronic kidney disease. We recommend an appropriate clinical evaluation that includes referral to a psychiatrist to evaluate insomnia and assess sleep hygiene in CKD patients.

Very often, the fundamental component of sleep hygiene is neglected, and relatively minor changes in patient behavior can lead to significant improvement in sleep quality. When this approach doesn't work clinicians should refer patients with CKD along with insomnia for cognitive behavioral therapy or medical therapy. Even though insomnia is prevalent among renal patients, nephrologists should be motivated to use the expertise of colleagues trained in sleep medicine and employ a multidisciplinary approach.

\section{Conclusions}

In patients with ESRD, the diagnosis and management of insomnia is overlooked due to an overlapping presentation with CKD. The approach to reviewing this association is to consider sleep disorders as a secondary consequence of the primary disease. Another method is to segregate insomnia from concurrent medical/psychiatric problems and manage it as an independent co-occurring disorder. The high proportion of insomnia in the CKD population highlights the need to conduct extensive and well-organized clinical trials. Furthermore, multidisciplinary and multifactorial research would explain the complex interrelationship between sleep and kidney disease. Consequently, this will lead to the development of novel treatments for sleep abnormalities, which can reduce the psychological and physiological burdens of CKD patients on HD.

\section{Additional Information \\ Disclosures}

Human subjects: Consent was obtained by all participants in this study. Medical Ethics Review Board of Khyber Teaching Hospital, Peshawar, Pakistan issued approval NEPH514/25/6/19. Animal subjects: All authors have confirmed that this study did not involve animal subjects or tissue. Conflicts of interest: In compliance with the ICMJE uniform disclosure form, all authors declare the following: Payment/services info: All authors have declared that no financial support was received from any organization for the submitted work. Financial relationships: All authors have declared that they have no financial relationships at present or within the previous three years with any organizations that might have an interest in the submitted work. Other relationships: All authors have declared that there are no other relationships or activities that could appear to have influenced the submitted work.

\section{References}

1. Stevens PE, Levin A: Evaluation and management of chronic kidney disease; synopsis of the kidney disease: improving global outcomes 2012 clinical practice guideline. Ann Intern Med. 2013, 158:825-830. 10.7326/0003-4819-158-11-201306040-00007

2. Nigam G, Camacho M, Chang ET, Riaz M: Exploring sleep disorders in patients with chronic kidney disease . Nat Sci Sleep. 2018, 10:35-43. 10.2147/NSS.S125839

3. Sateia MJ: International classification of sleep disorders-third edition: highlights and modifications . Chest. 2014, 146:1387-1394. 10.1378/chest.14-0970

4. Roth T: Insomnia: definition, prevalence, etiology, and consequences . J Clin Sleep Med. 2007, 3:5. $10.5664 /$ jcsm. 26929

5. Maung SC, El Sara A, Chapman C, Cohen D, Cukor D: Sleep disorders and chronic kidney disease . World J Nephrol. 2016, 5:224-232. 10.5527/wjn.v5.i3.224

6. Hanly P: Sleep disorders and end-stage renal disease . Curr Opin Pulm Med. 2008, 14:543-550. 10.1097/MCP.0b013e3283130f96

7. Aggarwal HK, Jain D, Dabas G, Yadav RK: Prevalence of depression, anxiety and insomnia in chronic kidney disease patients and their co-relation with the demographic variables. Pril (Makedon Akad Nauk Umet Odd Med Nauki). 2017, 38:35-44. 10.1515/prilozi-2017-0020

8. Scherer JS, Combs SA, Brennan F: Sleep disorders, restless legs syndrome, and uremic pruritus: diagnosis and treatment of common symptoms in dialysis patients. Am J Kidney Dis. 2017, 69:117-128. 10.1053/j.ajkd.2016.07.031

9. Sabbatini M, Minale B, Crispo A, et al.: Insomnia in maintenance haemodialysis patients . Nephrol Dial Transplant. 2002, 17:852-856. 10.1093/ndt/17.5.852

10. Rayner HC: Orexin as a possible cause of insomnia in dialysis patients . Am J Kidney Dis. 2003, 41:13351336. 10.1016/s0272-6386(03)00516-X

11. Marcus JN, Aschkenasi CJ, Lee CE, Chemelli RM, Saper CB, Yanagisawa M, Elmquist JK: Differential expression of orexin receptors 1 and 2 in the rat brain. J Comp Neurol. 2001, 435:6-25. 10.1002/cne.1190

12. Minisola S, Pepe J, Piemonte S, Cipriani C: The diagnosis and management of hypercalcaemia. BMJ. 2015, 


\section{Cureus}

350:h2723. 10.1136/bmj.h2723

13. Thorpy MJ: Classification of sleep disorders. Neurotherapeutics. 2012, 9:687-701. 10.1007/s13311-0120145-6

14. Fernandez-Mendoza J, Calhoun SL, Bixler EO, et al.: Sleep misperception and chronic insomnia in the general population: role of objective sleep duration and psychological profiles. Psychosom Med. 2011, 73:88-97. 10.1097/PSY.0b013e3181fe365a

15. Murtagh FE, Addington-Hall J, Higginson IJ: The prevalence of symptoms in end-stage renal disease: a systematic review. Adv Chronic Kidney Dis. 2007, 14:82-99. 10.1053/j.ackd.2006.10.001

16. Weisbord SD, Fried LF, Mor MK, et al.: Renal provider recognition of symptoms in patients on maintenance hemodialysis. Clin J Am Soc Nephrol. 2007, 2:960-967. 10.2215/CJN.00990207

17. Ahmad S, Gupta M, Gupta R, Dhyani M: Prevalence and correlates of insomnia and obstructive sleep apnea in chronic kidney disease. N Am J Med Sci. 2013, 5:641-646. 10.4103/1947-2714.122306

18. Coresh J, Selvin E, Stevens LA, et al.: Prevalence of chronic kidney disease in the United States . JAMA. 2007, 298:2038-2047. 10.1001/jama.298.17.2038

19. Sabry AA, Abo-Zenah H, Wafa E, et al.: Sleep disorders in hemodialysis patients. Saudi J Kidney Dis Transpl. 2010, 21:300-305.

20. Hamzi MA, Hassani K, Asseraji M, El Kabbaj D: Insomnia in hemodialysis patients: a multicenter study from Morocco. Saudi J Kidney Dis Transpl. 2017, 28:1112-1118. 10.4103/1319-2442.215152

21. Rai M, Rustagi T, Rustagi S, Kohli R: Depression, insomnia and sleep apnea in patients on maintenance hemodialysis. Indian J Nephrol. 2011, 21:223-229. 10.4103/0971-4065.83028

22. Tanaka K, Morimoto N, Tashiro N, Hori K, Katafuchi R, Fujimi S: The features of psychological problems and their significance in patients on hemodialysis--with reference to social and somatic factors. Clin Nephrol. 1999, 51:161-176.

23. Russcher M, Koch BC, Nagtegaal JE, et al.: Long-term effects of melatonin on quality of life and sleep in haemodialysis patients (Melody study): a randomized controlled trial. Br J Clin Pharmacol. 2013, 76:668679. 10.1111/bcp.12093

24. Yang B, Xu J, Xue Q, et al.: Non-pharmacological interventions for improving sleep quality in patients on dialysis: systematic review and meta-analysis. Sleep Med Rev. 2015, 23:68-82. 10.1016/j.smrv.2014.11.005 